\title{
Periodic Orbits of the First Kind in the Autonomous Four-body Problem with the Case of Collision
}

\author{
M. R. Hassan1, Md. Aminul Hassan², Payal Singh'3, Vinay Kumar', R. R. Thapa5 \\ ${ }^{1}$ Department of Mathematics, S. M. College, T. M. Bhagalpur University, Bhagalpur, India \\ ${ }^{2}$ GTE, Bangalore, India \\ ${ }^{3}$ Research Scholar, T. M. Bhagalpur University, Bhagalpur, India \\ ${ }^{4}$ Department of Mathematics, Zakir Hussain College, University of Delhi, New Delhi, India \\ ${ }^{5}$ Department of Mathematics, P. G. Campus, Tribhuvan University, Biratnagar, Nepal \\ Email: hassansmc@gmail.com,mahassan012@gmail.com
}

How to cite this paper: Hassan, M.R. Hassan, Md. A., Singh, P., Kumar, V. and Thapa, R.R. (2017) Periodic Orbits of the First Kind in the Autonomous Four-body Problem with the Case of Collision. International Journal of Astronomy and Astrophysics, 7, 91-111.

https://doi.org/10.4236/ijaa.2017.72008

Received: April 1, 2017

Accepted: June 9, 2017

Published: June 12, 2017

Copyright (C) 2017 by authors and Scientific Research Publishing Inc. This work is licensed under the Creative Commons Attribution International License (CC BY 4.0).

http://creativecommons.org/licenses/by/4.0/

\begin{abstract}
In this manuscript, the existence of periodic orbits of collision of the first kind has been discussed on the model of Autonomous Four-body Problem by the method of analytic continuation given by Giacaglia [1] and Bhatnagar [2] [3]. For the existence of periodic orbits, Duboshin's criterion [4] has been satisfied and it has been confirmed by analyzing the Poincare surfaces of section (PSS) [5]. Also it has been shown that the case of collision given by Levi-Civita [6] [7] is conserved by the method analytic continuation. In all sections of this manuscript, equilateral triangular configuration given by Ceccaroni and Biggs [8] has been considered. In this model, third primary of inferior mass (in comparison of the other primaries) is placed at the equilibrium point $L_{4}$ of the R3BP.
\end{abstract}

\section{Keywords}

Autonomous Four-Body Problem, Regularization, Periodicity, Poincare Surfaces of Section, Collision Orbit, Zero Velocity Curves

\section{Introduction}

We know that the four most popular methods of proving the existence of periodic orbits are:

(i) the method of analytic continuation,

(ii) the process of equating Fourier coefficients of equal frequencies,

(iii) the application of fixed point theorem given by Poincare, 
(iv) the method of power series.

Giacaglia [1] used the method of analytic continuation to examine the existence of periodic orbits of collision in the Restricted Three-body Problem (R3BP). Bhatnagar [2] generalized the problem in elliptic case. The problem of Giacaglia [1] was further extended by Bhatnagar [3] in the R4BP by taking the primaries at the vertices of an equilateral triangle. With different perturbations like oblateness, triaxiality, photogravitation, Pointing-Robertson drag effects of the primaries, the existence of periodic orbits of collision in the R3BP and in the R4BP, have been studied by different authors in two and three-dimensional co-ordinate system during the period of last three decades of the $20^{\text {th }}$ century but nobody established the proper mathematical model of the R4BP. Recently Ceccaroni and Biggs [8] has studied the autonomous coplanar CR4BP by taking the third primary of comparatively inferior mass at the triangular equilibrium point $L_{4}$ of R3BP and with an extension to low-thrust propulsion for application to the future science mission.

In present paper, we have proposed to study the existence of periodic orbits of first kind in the Autonomous Four-body Problem by the method of analytic continuation. By using Poincare surfaces of section (PSS), the conditions for the existence of periodic orbits given by Duboshin [4] have been confirmed. For collision case, we have applied the criterion given by Levi-Civitas [6] [7] and it is satisfied by our model.

\section{Equations of Motion}

Let $P_{1}, P_{2}, P_{3}$ be the three massive bodies of masses $m_{i}(i=1,2,3)$ respectively, where $m_{1} \geq m_{2} \gg m_{3}$ and the fourth body of mass $m$ be at $P$. These bodies are moving in the same plane under some restrictions as follows:

The fourth body at $P$ of mass $m$ is assumed to be of infinitesimal mass not influencing the motion of $P_{1}, P_{2}, P_{3}$ but motions of $P_{(4)}$ is being influenced by the motions of $P_{1}, P_{2}, P_{3}$. Further, we have assumed that the mass $m_{3}$ at $P_{3}$ is taken small enough, so that it can't influence the motion of the dominating primaries $P_{1}$ and $P_{2}$ and it is placed at any one of the triangular libration points (Lagrangian Points) of the classical restricted three body problem. Since the third primary can't influence the motions of $P_{1}$ and $P_{2}$, so the centre of rotation of the system remains at the barycentre of two main primaries $P_{1}$ and $P_{2}$. Also, it is supposed, all the primaries are moving in the same plane in circular orbits around the bary-centre of massive primaries $P_{1}$ and $P_{2}$ with the same angular velocity $\omega$ and the fourth body $P_{4}$ is moving under the gravitational field and plane of motion of three primaries $P_{1}, P_{2}, P_{3}$ then to check the nature of motion of infinitesimal mass $m_{4}$.

Let the line joining $P_{1}$ and $P_{2}$ be taken as the $x$-axis and their mass centre (bary-centre) $O$, as the origin. Let the line through $O$ and perpendicular to $P_{1} P_{2}$ lying in the plane of motion of the primaries be taken as the $y$-axis. Let the positions of masses $m_{i}(i=1,2,3)$ be $P_{1}\left(x_{1}, 0\right), P_{2}\left(x_{2}, 0\right), P_{3}\left(x_{3}, y_{3}\right)$ and $P(x, y)$ respectively. Let $\boldsymbol{r}$ be the position vector of $P$ and $\boldsymbol{r}_{1}, \boldsymbol{r}_{2}, \boldsymbol{r}_{3}$ be the displace- 


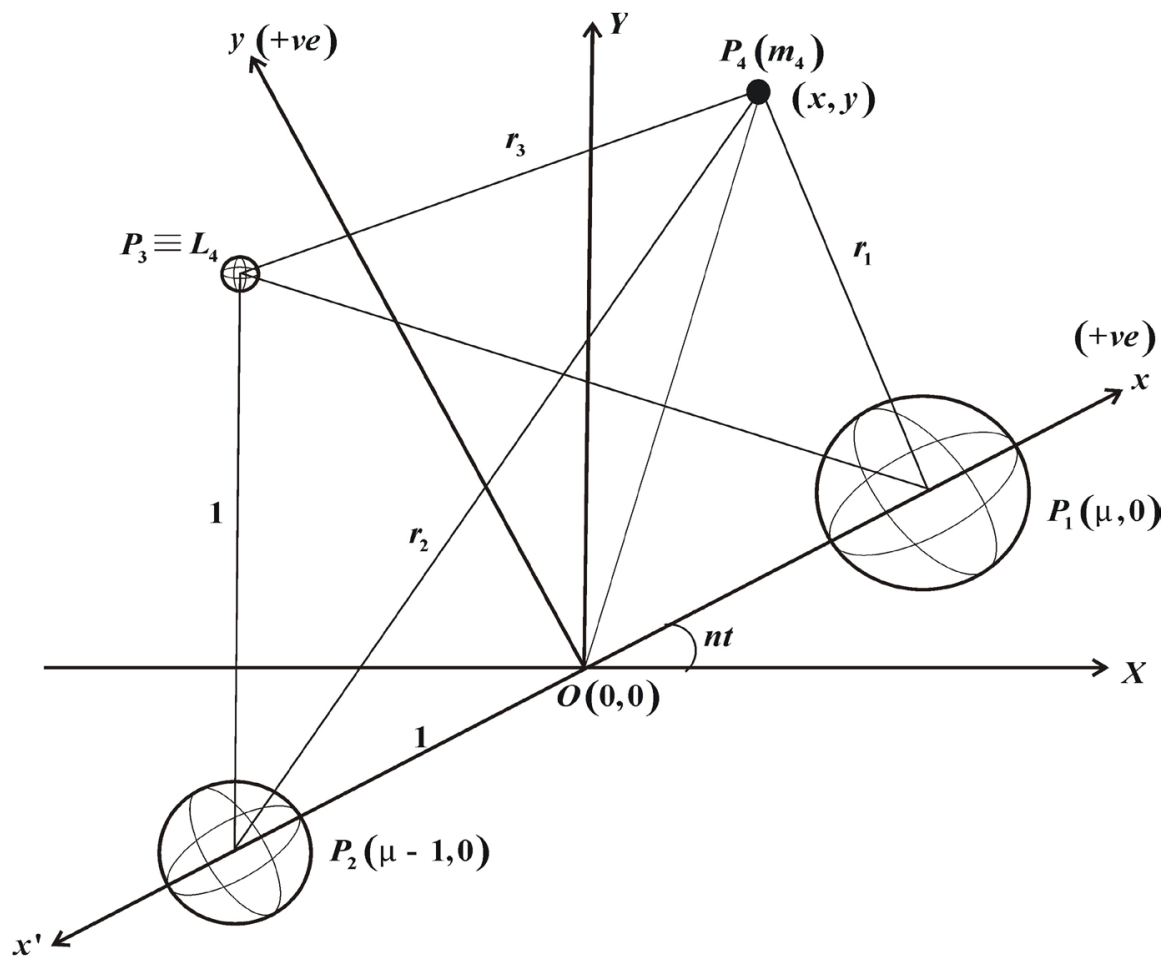

Figure 1. Configuration of four-body problem.

ments of $P_{1}, P_{2}$ and $P_{3}$ relative to $P_{4}$ as shown in Figure 1, then

$$
\left.\begin{array}{l}
\boldsymbol{r}=x \hat{i}+y \hat{j}, \\
\boldsymbol{r}_{1}=\left(x-x_{1}\right) \hat{i}+y \hat{j}, \\
\boldsymbol{r}_{2}=\left(x-x_{2}\right) \hat{i}+y \hat{j}, \\
\boldsymbol{r}_{3}=\left(x-x_{3}\right) \hat{i}+\left(y-y_{3}\right) \hat{j} .
\end{array}\right\}
$$

Let $\boldsymbol{F}_{1}, \boldsymbol{F}_{2}, \boldsymbol{F}_{3}$ be the gravitational forces exerted on $P_{4}\left(m_{4}\right)$ by the primaries respectively, then

$$
\boldsymbol{F}_{i}=\gamma m_{i} m \frac{\boldsymbol{r}_{i}}{r_{i}^{3}}(i=1,2,3),
$$

where $\gamma$ is the gravitational constant.

The total gravitational force acting on $P(m)$ by the three primaries is given by

$$
\boldsymbol{F}=\sum_{i=1}^{3} F_{i}=\gamma m_{4} \sum_{i=1}^{3} \frac{m_{i} \boldsymbol{r}_{i}}{r_{i}^{3}} .
$$

Let $n$ be the magnitude of angular velocity $\omega$ and $\hat{k}$ be the unit vector normal to the plane of motion of the primaries, then $\omega=n \hat{k}$.

The Equation of motion of the infinitesimal mass $m_{4}$ in synodic frame is

$$
m_{4}\left[\frac{\partial^{2} \boldsymbol{r}}{\partial t_{2}}+2 \boldsymbol{\omega} \times \frac{\partial \boldsymbol{r}}{\partial t}+\frac{\partial \boldsymbol{\omega}}{\partial t} \times \boldsymbol{r}+\boldsymbol{\omega} \times(\boldsymbol{\omega} \times \boldsymbol{r})\right]=\boldsymbol{F} .
$$

Since the synodic frame are revolving with constant angular velocity about the bary-centre, hence $\frac{\partial \boldsymbol{\omega}}{\partial t}=0$ and thus Equation (4) reduces to 


$$
\left[\frac{\partial^{2} \boldsymbol{r}}{\partial t^{2}}+2 \boldsymbol{\omega} \times \frac{\partial \boldsymbol{r}}{\partial t}+\boldsymbol{\omega} \times(\boldsymbol{\omega} \times \boldsymbol{r})\right]=\gamma \sum_{i=1}^{3} \frac{m_{i} \boldsymbol{r}_{i}}{r_{i}^{3}}
$$

In cartesian form, the equations of motion of the infinitesimal mass $m_{4}$ in the gravitational field of three primaries, are given by

$$
\left.\begin{array}{l}
\ddot{x}-2 n \dot{y}-n^{2} x=\gamma\left[\frac{m_{1}\left(x-x_{1}\right)}{r_{1}^{3}}+\frac{m_{2}\left(x-x_{2}\right)}{r_{2}^{3}}+\frac{m_{3}\left(x-x_{3}\right)}{r_{3}^{3}}\right], \\
\ddot{y}+2 n \dot{x}-n^{2} y=\gamma\left[\frac{m_{1} y}{r_{1}^{3}}+\frac{m_{2} y}{r_{2}^{3}}+\frac{m_{3}\left(y-y_{3}\right)}{r_{3}^{3}}\right] .
\end{array}\right\}
$$

Also the linear velocity of the infinitesimal mass $m$ on its orbit; is given by

$$
\boldsymbol{v}=\frac{\mathrm{d} \boldsymbol{r}}{\mathrm{d} t}=\frac{\partial \boldsymbol{r}}{\partial t}+\boldsymbol{\omega} \times \boldsymbol{r} . \quad\left[\text { As } \frac{\mathrm{d}}{\mathrm{d} t}=\frac{\partial}{\partial t}+\boldsymbol{\omega} \times\right]
$$

If $v_{1}, v_{2}$ are two components of $\boldsymbol{v}$, then from Equation (7),

$$
v_{1}=\dot{x}-n y, \quad v_{2}=\dot{y}-n x .
$$

If mass of the infinitesimal body is supposed to be unity, then the kinetic energy of the infinitesimal mass is given by

$$
T=\frac{1}{2}\left(v_{1}^{2}+v_{2}^{2}\right)=\frac{1}{2}\left[\left(\dot{x}^{2}+\dot{y}^{2}\right)+2 n(x \dot{y}-\dot{x} y)+n^{2}\left(x^{2}+y^{2}\right)\right],
$$

Let $p_{1}, p_{2}$ be the momenta corresponding to the co-ordinates $x, y$ respectively, then

$$
p_{1}=\frac{\partial T}{\partial \dot{x}}=(\dot{x}-n y)=v_{1}, \quad p_{2}=\frac{\partial T}{\partial \dot{y}}=(\dot{y}+n x)=v_{2} .
$$

Combination of Equations ((9) and (10)) yields

$$
T=\frac{1}{2}\left(p_{1}^{2}+p_{2}^{2}\right) .
$$

The gravitational potential of the body of mass $m_{i}$ at any point of $P_{4}$ outside it, is given by

$$
V_{i}=-\frac{\gamma m_{i}}{r_{i}}(i=1,2,3)
$$

then, total gravitational potential at $P_{4}$ due to three primaries is given by

$$
V=\sum_{i=1}^{3} V_{i}=-\gamma\left(\frac{m_{1}}{r_{1}}+\frac{m_{2}}{r_{2}}+\frac{m_{3}}{r_{3}}\right) .
$$

The Hamiltonian of the infinitesimal body of unit mass is given by

$$
\begin{gathered}
H=p_{1} \dot{x}+p_{2} \dot{y}-(T-V), \\
H=\frac{1}{2}\left(p_{1}^{2}+p_{2}^{2}\right)+n\left(p_{1} y-p_{2} x\right)-\gamma\left(\frac{m_{1}}{r_{1}}+\frac{m_{2}}{r_{2}}+\frac{m_{3}}{r_{3}}\right)=C .
\end{gathered}
$$

Let $\mu$ be the reduced mass of the second primary and $\varepsilon$ be the reduced mass of the third primary, then from the definition of reduced mass, we have

$$
\frac{m_{2}}{m_{1}+m_{2}}=\mu, \frac{m_{3}}{m_{1}+m_{2}}=\varepsilon \text { and } m_{1}+m_{2}=1 .
$$


then $m_{1}=1-\mu, m_{2}=\mu, m_{3}=\varepsilon$.

The coordinates of $P_{1}, P_{2}, P_{3}$ are given by

$$
(\mu, 0),(\mu-1,0) \text { and } P_{3}\left(x_{3}, y_{3}\right) \equiv L_{4}\left(\mu-\frac{1}{2}, \frac{\sqrt{3}}{2}\right) \text {. }
$$

Clearly $\left|P_{1} P_{2}\right|=\left|P_{2} P_{3}\right|=\left|P_{3} P_{1}\right|=1$, which implies that $P_{1} P_{2} P_{3}$ forms an equilateral triangle of sides of unit length. We know that $\varepsilon$ is very small in comparison of masses of the other two primaries, so we can choose $\varepsilon$ as the order of $\mu$ i.e., $\varepsilon=o(\mu)=\varepsilon_{0} \mu$ (say). Now choosing unit of time in such a manner that $\gamma=1$ and $n=1$ and taking $x=x_{1}, y=x_{2}$, then the Hamilton canonical equations of motion of the infinitesimal body $P_{4}$ are given by

$$
\frac{\mathrm{d} x_{i}}{\mathrm{~d} t}=\frac{\partial H}{\partial p_{i}}, \frac{\mathrm{d} p_{i}}{\mathrm{~d} t}=-\frac{\partial H}{\partial x_{i}}(i=1,2),
$$

where

$$
H=\frac{1}{2}\left(p_{1}^{2}+p_{2}^{2}\right)+\left(p_{1} x_{2}-p_{2} x_{1}\right)-\frac{1-\mu}{r_{1}}-\frac{\mu}{r_{2}}-\frac{\varepsilon_{0} \mu}{r_{3}}=C
$$

is the reduced Hamiltonian corresponding to canonically conjugate variables $\left(x_{1}, x_{2}\right)$ and $\left(p_{1}, p_{2}\right)$.

\section{Regularization at the Singularity $\left(r_{1}=0\right)$}

In our Hamiltonian $H$ given in Equation (15), there are three singularities $r_{1}=r_{2}=r_{3}=0$. To examine the existence of periodic orbits of collision with the first primary, we have to eliminate the singularity $r_{1}=0$. For this, let us define an extended generating function $S$ given by

$$
S=\left(\mu+q_{1}^{2}-q_{2}^{2}\right) p_{1}+\left(2 q_{1} q_{2}\right) p_{2},
$$

with

$$
x_{i}=\frac{\partial S}{\partial p_{i}}, \quad Q_{i}=\frac{\partial S}{\partial q_{i}}, \quad(i=1,2)
$$

where $Q_{i}$ is the momenta associated with new co-ordinate $q_{i}(i=1,2)$.

Clearly,

$$
\left.\begin{array}{l}
x_{1}=\frac{\partial S}{\partial p_{1}}=\mu+q_{1}^{2}-q_{2}^{2}, x_{2}=\frac{\partial S}{\partial p_{1}}=2 q_{1} q_{2} . \\
r_{1}=q_{1}^{2}+q_{2}^{2}, \\
r_{2}^{2}=1+2\left(q_{1}^{2}-q_{2}^{2}\right)^{2}+\left(q_{1}^{2}+q_{2}^{2}\right)^{2}, \\
r_{3}^{2}=1+\left(q_{1}^{2}-q_{2}^{2}\right)^{2}-2 \sqrt{3} q_{1} q_{2}+\left(q_{1}^{2}+q_{2}^{2}\right)^{2} .
\end{array}\right\}
$$

Also,

$$
\left.\begin{array}{ll}
Q_{1}=2\left(q_{1} p_{1}+q_{2} p_{2}\right), & Q_{2}=2\left(q_{1} p_{2}-q_{2} p_{1}\right), \\
p_{1}=\frac{1}{2 r_{1}}\left(Q_{1} q_{1}-Q_{2} q_{2}\right), & p_{2}=\frac{1}{2 r_{1}}\left(Q_{1} q_{2}+Q_{2} q_{1}\right) .
\end{array}\right\}
$$


Thus the Hamiltonian $H$ given in Equation (15), can be written in terms of new variables $Q_{i}, q_{i}$, as

$H=\frac{1}{8 r_{1}}\left(Q_{1}^{2}+Q_{2}^{2}\right)+\frac{1}{2}\left(Q_{1} q_{2}-Q_{2} q_{1}\right)-\frac{\mu}{2 r_{1}}\left(Q_{1} q_{2}+Q_{2} q_{1}\right)-\frac{1-\mu}{r_{1}}-\frac{\mu}{r_{2}}-\frac{\varepsilon_{0} \mu}{r_{3}}=C$.

Let us introduce pseudo time $\tau$ by the differential equation

$$
\mathrm{d} t=r_{1} \mathrm{~d} \tau(\tau=0 \text { when } t=0) .
$$

Thus the regularized Hamilton-canonical equations of motion of the infinitesimal body corresponding to the Hamiltonian $K=0$, are given by

$$
\frac{\mathrm{d} q_{i}}{\mathrm{~d} \tau}=\frac{\partial K}{\partial Q_{i}}, \quad \frac{\mathrm{d} Q_{i}}{\mathrm{~d} \tau}=-\frac{\partial K}{\partial q_{i}}(i=1,2),
$$

where the regularized Hamiltonian $K$ is given by

$$
\begin{gathered}
K=r_{1}(H-C)=0, \\
K=\frac{1}{8}\left(Q_{1}^{2}+Q_{2}^{2}\right)+\frac{1}{2} r_{1}\left(Q_{1} q_{2}-Q_{2} q_{1}-2 C_{0}\right)-1 \\
+\mu\left[1-\frac{1}{2}\left(Q_{1} q_{2}+Q_{2} q_{1}\right)-r_{1} C_{1}-\frac{r_{1}}{r_{2}}-\frac{r_{1} \varepsilon_{0}}{r_{3}}\right]=0 .
\end{gathered}
$$

Let us write $K=K_{0}+\mu K_{1}$, then

$$
\begin{gathered}
K_{0}=\frac{1}{8}\left(Q_{1}^{2}+Q_{2}^{2}\right)+\frac{1}{2} r_{1}\left(Q_{1} q_{2}-Q_{2} q_{1}-2 C_{0}\right)-1=-\lambda, \text { (say) } \\
K_{1}=1-\frac{1}{2}\left(Q_{1} q_{2}+Q_{2} q_{1}\right)-r_{1} C_{1}-\frac{r_{1}}{r_{2}}-\frac{r_{1} \varepsilon_{0}}{r_{3}} .
\end{gathered}
$$

\section{Generating Solution (i.e., Solutions When $\mu=0$ )}

For generating solutions, we shall choose $K_{0}$ for our Hamiltonian function, so in order to solve the Hamilton-Jacobi equation associated with $K_{0}$, let us write

$$
Q_{i}=\frac{\partial W}{\partial q_{i}}, i=1,2 \text { and } 1-\lambda=\alpha>0,
$$

where $\alpha$ is an arbitrary constant.

Since $t$ is not involved explicitly in $K_{0}$ : hence by using Equation (27) in Equation (25), the Hamilton-Jacobi equation may be written as

$$
\frac{1}{8}\left[\left(\frac{\partial W}{\partial q_{1}}\right)^{2}+\left(\frac{\partial W}{\partial q_{2}}\right)^{2}\right]+\frac{r_{1}}{2}\left[q_{2} \frac{\partial W}{\partial q_{1}}-q_{1} \frac{\partial W}{\partial q_{2}}-2 C_{0}\right]=\alpha,
$$

Putting $q_{1}=\rho \cos \varphi, q_{2}=\rho \sin \varphi$, then the Equation (27) becomes

$$
\frac{1}{8}\left[\left(\frac{\partial W}{\partial \rho}\right)^{2}+\frac{1}{\rho^{2}}\left(\frac{\partial W}{\partial \varphi}\right)^{2}\right]+\frac{1}{2} \rho^{2}\left[-\frac{\partial W}{\partial \varphi}-2 C_{0}\right]=\alpha .
$$

It may be noted that this differential equation is exactly the same as in Giacaglia [1] and Bhatnagar [2] [3] and therefore the solution of Equation (29) can be written by the method of separation of variables, as

$$
W=U(\rho)+2 G \varphi,
$$


where $G$ is an arbitrary constant.

Let us introduce a new quantity $z$ by $r_{1}=\rho^{2}=z$ then from Equation (30), we get

$$
\frac{\partial W}{\partial \rho}=\frac{\partial U}{\partial \rho}=\frac{\mathrm{d} U}{\mathrm{~d} \rho}=2 \rho \frac{\mathrm{d} U}{\mathrm{~d} z} \text { and } \frac{\partial W}{\partial \rho}=2 G
$$

Combination of Equations (29) and (30) yields

$$
\begin{gathered}
\frac{1}{8}\left[\left(2 \rho \frac{\mathrm{d} U}{\mathrm{~d} z}\right)^{2}+\left(\frac{2 G}{\rho}\right)^{2}\right]+\frac{\rho^{2}}{\mathrm{z}}\left[-2 G-2 C_{0}\right]=\alpha, \\
\text { i.e., } z\left(\frac{\mathrm{d} U}{\mathrm{~d} z}\right)^{2}+\frac{G^{2}}{z}-2 z\left(G+C_{0}\right)=2 \alpha, \\
\frac{\mathrm{d} U}{\mathrm{~d} z}=\left[-2\left(G+C_{0}\right)\right]^{\frac{1}{2}} \frac{\sqrt{f(z)}}{z},
\end{gathered}
$$

where

$$
\begin{gathered}
f(z)=-z^{2}-\frac{\alpha z}{G+C_{0}}+\frac{G^{2}}{2\left(G+C_{0}\right)}, \\
U(z, G, \alpha)=\left[-2\left(G+C_{0}\right)\right]^{\frac{1}{2}} \int_{z_{1}}^{z} \frac{\sqrt{f(z)}}{z} \mathrm{~d} z,
\end{gathered}
$$

where $z_{1}$ is the smaller root of the roots of the equation $f(z)=0$.

From Equation (33), we conclude that for general solution; we need only two arbitrary constants as $\alpha$ and $G$. Therefore the solution of Equation (30) may be regarded as a general solution.

Let us introduce the parameters $a, e, l$ by the relations

$$
\left.\begin{array}{rl}
Z_{1} & =a(1-e), \quad z_{2}=a(1+e), \\
Z & =z_{1} \cos ^{2}\left(\frac{l}{2}\right)+z_{2} \sin ^{2}\left(\frac{l}{2}\right), \quad(0 \leq e \leq 1) \\
& =a(1-e \cos l),
\end{array}\right\}
$$

where $a$ is the semi-major axis, $e$ is the eccentricity and $l$ is the latus-rectum of the elliptic orbit of the infinitesimal body.

It may be noted that for $z=z_{1}, l=0$ and $z_{2}$ is the other root of the equation $f(z)=0$.

We introduce a parameter $L$ by the relation

$$
\begin{aligned}
& \alpha=L\left[-2\left(G+C_{0}\right)\right]^{\frac{1}{2}}>0, \quad \text { so that; } \\
& a=\frac{z_{1}+z_{2}}{2}=\frac{L}{\left[\sqrt{-2\left(G+C_{0}\right)}\right]^{\frac{1}{2}}}>0 \\
& e=\left(1-\frac{z_{1} z_{2}}{a^{2}}\right)^{\frac{1}{2}}=\left(\sqrt{1-\frac{G^{2}}{L^{2}}}\right)^{\frac{1}{2}} \leq 1
\end{aligned}
$$

From Equations (33), (35) and (36), we get 


$$
\begin{gathered}
f(z)=-z^{2}-\frac{z L\left[-2\left(G+C_{0}\right)\right]^{\frac{1}{2}}}{G+C_{0}}+\frac{G^{2}}{2\left(G+C_{0}\right)} \\
=-z^{2}+2 a z+\frac{G^{2}}{2\left(G+C_{0}\right)}=-z^{2}+2 a z-\frac{a^{2} G^{2}}{L^{2}} \\
=-a^{2}(1-e \cos l)^{2}+2 a^{2}(1-e \cos l)+a^{2}\left(e^{2}-1\right), \\
\text { i.e., } f(z)=a^{2} e^{2} \sin ^{2} l .
\end{gathered}
$$

Again from Equation (25)

$$
\begin{aligned}
& K_{0}=\frac{1}{8}\left(Q_{1}^{2}+Q_{2}^{2}\right)+\frac{\rho^{2}}{2}\left(Q_{1} q_{2}-Q_{2} q_{1}-2 C_{0}\right)-1, \\
& \frac{\partial K_{0}}{\partial Q_{1}}=\frac{1}{4} Q_{1}+\frac{1}{2} \rho^{2} q_{2}, \frac{\partial K_{0}}{\partial Q_{2}}=\frac{1}{4} Q_{2}-\frac{1}{2} \rho^{2} q_{1} .
\end{aligned}
$$

Thus the equations of motion associated with $K_{0}$ are given as

$$
\begin{gathered}
\frac{\mathrm{d} q_{1}}{\mathrm{~d} \tau}=\frac{\partial K_{0}}{\partial Q_{1}}=\frac{1}{4} Q_{1}+\frac{1}{2} \rho^{2} q_{2}, \quad \frac{\mathrm{d} q_{2}}{\mathrm{~d} \tau}=\frac{\partial K_{0}}{\partial Q_{2}}=\frac{1}{4} Q_{2}-\frac{1}{2} \rho^{2} q_{1}, \\
\text { i.e., } q_{1}^{\prime}=\frac{1}{4} Q_{1}+\frac{1}{2} \rho^{2} q_{2}, \quad q_{2}^{\prime}=\frac{1}{4} Q_{2}+\frac{1}{2} \rho^{2} q_{1},
\end{gathered}
$$

where (') denotes the differentiation with respect to $\tau$.

Now from $z=\rho^{2}$ we get $\frac{\mathrm{d} z}{\mathrm{~d} \tau}=2 \rho \frac{\mathrm{d} \rho}{\mathrm{d} \tau}=2 \rho \rho^{\prime}$.

Also $\rho^{2}=q_{1}^{2}+q_{2}^{2}$ implies $\rho \rho^{\prime}=q_{1} q_{1}^{\prime}+q_{2} q_{2}^{\prime}=\sum_{i=1}^{2} q_{i} q_{i}^{\prime}$

and $\sum_{i=1}^{2} q_{i} Q_{i}=q_{1} Q_{1}+q_{2} Q_{2}=4 \sum q_{i} q_{i}^{\prime}$. [Using Equation (38)]

Thus from the above relations, we have

$$
\frac{1}{2} \frac{\mathrm{d} z}{\mathrm{~d} \tau}=\rho \rho^{\prime}=\sum_{i=1}^{2} q_{i} q_{i}^{\prime}=\frac{1}{4} \sum_{i=1}^{2} q_{i} Q_{i}^{\prime}=\frac{z}{2} \frac{\mathrm{d} U}{\mathrm{~d} z} .
$$

From Equation (32), we get

$$
\begin{aligned}
& z \frac{\mathrm{d} U}{\mathrm{~d} z}=\left[-2\left(G+C_{0}\right)\right]^{\frac{1}{2}} \sqrt{f(z)}, \\
& \frac{\mathrm{d} z}{\mathrm{~d} \tau}=\left[-2\left(G+C_{0}\right)\right]^{\frac{1}{2}} \sqrt{f(z)}, \quad \text { [Using Equation (39)] } \\
& \frac{\mathrm{d} z}{\sqrt{f(z)}}=\left[-2\left(G+C_{0}\right)\right]^{\frac{1}{2}} \mathrm{~d} \tau, \\
& \text { i.e., } \int_{z_{1}}^{z} \frac{\mathrm{d} z}{\sqrt{f(z)}}=\left[-2\left(G+C_{0}\right)\right]^{\frac{1}{2}} \int_{0}^{\tau} \mathrm{d} \tau, \\
& \text { i.e., } l=\left[-2\left(G+C_{0}\right)\right]^{\frac{1}{2}}\left(\tau-\tau_{0}\right) .
\end{aligned}
$$

From Equation (30),

$$
\begin{aligned}
& W=U(\rho)+2 G \varphi, \\
& \frac{\partial W}{\partial G}=\frac{\partial U}{\partial G}+2 \varphi,
\end{aligned}
$$


where $\frac{\partial U}{\partial G}=\frac{\partial}{\partial G} U(z, G, \alpha)$,

$$
\begin{gathered}
\frac{\partial U}{\partial G}=\frac{\partial}{\partial G}\left[-2\left(G+C_{0}\right)\right]^{\frac{1}{2}} \int_{z_{1}}^{z} \frac{\sqrt{f(z)}}{z} \mathrm{~d} z, \quad[\text { Using Equation (34)] } \\
\frac{\partial U}{\partial G}=\frac{\sqrt{L^{2}-G^{2}}}{2\left(G+C_{0}\right)} \sin l-h,
\end{gathered}
$$

where $h=\sqrt{1-e^{2}} \int_{0}^{l} \frac{\mathrm{d} l}{1-e \cos l}$.

If we take $L$ and $G$ as arbitrary constants, the solutions may be written as

$$
\left.\begin{array}{l}
\frac{\partial W}{\partial L}=\frac{\partial U}{\partial L}=l, \\
\frac{\partial W}{\partial G}=2 \varphi+\frac{\sqrt{L^{2}-G^{2}}}{2\left(G+C_{0}\right)} \sin l-h=g \text { (say). }
\end{array}\right\}
$$

From the second equation of system (41), we get the argument as

$$
\left.\begin{array}{l}
\varphi=\frac{1}{2}(g+h)-\frac{\sqrt{L^{2}-G^{2}}}{4\left(G+C_{0}\right)} \sin l(e \neq 1) \\
\text { and } \varphi=\frac{1}{2} g-\frac{1}{4 C_{0}} \sin l \text { for }(e=1),(G=0),(h=0)
\end{array}\right\}
$$

Since

$$
K_{0}=\alpha-1=L\left[-2\left(G+C_{0}\right)\right]^{\frac{1}{2}}-1,
$$

hence for the problem generated by Hamiltonian $K_{0}$ (regularized two-body problem in rotating co-ordinate system), we have

$$
\begin{aligned}
& \frac{\mathrm{d} L}{\mathrm{~d} \tau}=-\frac{\partial K_{0}}{\partial l}=0, L=\text { const. }=L_{0} \\
& \frac{\mathrm{d} G}{\mathrm{~d} \tau}=-\frac{\partial K_{0}}{\partial g}=0, G=\text { const. }=G_{0} \\
& \frac{\mathrm{d} l}{\mathrm{~d} \tau}=\frac{\partial K_{0}}{\partial L}=\left[-2\left(G+C_{0}\right)\right]^{\frac{1}{2}}=\text { const. }=\eta_{l}, \\
& \text { where } l=\eta_{l} \tau+l_{0}, \\
& \frac{\mathrm{d} g}{\mathrm{~d} \tau}=\frac{\partial K_{0}}{\partial G}=\frac{-L}{\left[-2\left(G+C_{0}\right)\right]}=\text { const. }=\eta_{g}, \\
& \text { where } g=\eta_{g} \tau+g_{0} .
\end{aligned}
$$

The variables $q_{i}, Q_{i}(i=1,2)$ can now be expressed in terms of the canonical elements for $e \neq 1,(G \neq 0)$, as

$$
\left.\begin{array}{ll}
q_{1}= \pm[a(1-e \cos l)]^{\frac{1}{2}} \cos \varphi, & q_{2}= \pm[a(1-e \cos l)]^{\frac{1}{2}} \sin \varphi, \\
Q_{1}= \pm \frac{2 e L \sin l \cos \varphi-2 G \cos \varphi}{[a(1-e \cos l)]^{\frac{1}{2}}}, & Q_{2}= \pm \frac{2 e L \sin l \sin \varphi+2 G \cos \varphi}{[a(1-e \cos l)]^{\frac{1}{2}}}
\end{array}\right\}
$$

where $\varphi$ is given by the first equation of system (42). 
When $e=1$ and $G=0$, then

$$
\left.\begin{array}{l} 
\pm q_{1}=\sqrt{2 a} \sin \left(\frac{l}{2}\right) \cos \varphi, \quad \pm q_{2}=\sqrt{2 a} \sin \left(\frac{l}{2}\right) \sin \varphi, \\
\pm Q_{1}=\frac{4 L}{\sqrt{2 a}} \cos \left(\frac{l}{2}\right) \cos \varphi, \quad \pm Q_{2}=\frac{4 L}{\sqrt{2 a}} \cos \left(\frac{l}{2}\right) \sin \varphi,
\end{array}\right\}
$$

where $\varphi$ is given by the second equation of system (42).

The original synodic cartesian co-ordinates in a non-uniformly rotating system are obtained from Equations (18) and (20), when $\mu=0$, as

$$
\left.\begin{array}{ll}
x_{1}=q_{1}^{2}-q_{2}^{2}, & x_{2}=2 q_{1} q_{2} \\
p_{1}=\frac{q_{1} Q_{1}-q_{2} Q_{2}}{2 z}, & p_{2}=\frac{q_{1} Q_{2}+q_{2} Q_{2}}{2 Z} .
\end{array}\right\}
$$

The sidereal cartesian co-ordinates are obtained by considering the transformations

$$
\left.\begin{array}{l}
X_{1}=x_{1} \cos t-x_{2} \sin t, \quad X_{2}=x_{1} \sin t+x_{2} \cos t, \\
\dot{X}_{1}=p_{1} \cos t-p_{2} \sin t, \quad \dot{X}_{2}=p_{1} \sin t+p_{2} \cos t,
\end{array}\right\}
$$

where $t$ is given by

$$
\begin{gathered}
t=\int \frac{z \mathrm{~d} z}{\sqrt{-2\left(G+C_{0}\right) F(z)}}, \\
\text { i.e., } t-t_{0}=\frac{a}{\sqrt{-2\left(G+C_{0}\right)}}\{l-e \sin l\},
\end{gathered}
$$

where $t_{0}$ is a constant.

In terms of canonical variables introduced, the complete Hamiltonian may be written as $K=L\left[-2\left(G+C_{0}\right)\right]^{\frac{1}{2}}-1+\mu R$, where $R$ can be obtained from Equation (26) after changing into canonical variables.

The equations of motion for the complete Hamiltonian are

$$
\left.\begin{array}{l}
\frac{\mathrm{d} l}{\mathrm{~d} \tau}=\frac{\partial K}{\partial L}=\left[-2\left(G+C_{0}\right)\right]^{\frac{1}{2}}+\mu \frac{\partial R}{\partial L}, \\
\frac{\mathrm{d} g}{\mathrm{~d} \tau}=\frac{\partial K}{\partial G}=-\frac{L}{\left[-2\left(G+C_{0}\right)\right]^{\frac{1}{2}}}+\mu \frac{\partial R}{\partial G}, \\
\frac{\mathrm{d} L}{\mathrm{~d} \tau}=-\frac{\partial K}{\partial l}=-\mu \frac{\partial R}{\partial l}, \frac{\mathrm{d} G}{\mathrm{~d} \tau}=-\frac{\partial K}{\partial g}=-\mu \frac{\partial R}{\partial g} .
\end{array}\right\}
$$

Equation (49) forms the basis of a general perturbation theory for the present problem. The solution described by Equations ((44) and (45)) and is periodic if $l$ and $g$ have commensurable frequencies, i.e., if

$$
\left|\frac{\eta_{l}}{\eta_{g}}\right|=\frac{2\left(G+C_{0}\right)}{L}=\frac{p}{q},
$$

where $p$ and $q$ are integers.

The periods of $\left|q_{i}, Q_{i}\right|$ are $\frac{4 \pi}{n_{l}}$ and $\frac{4 \pi}{n_{g}}$ respectively, so that in case of 
commensurability, the period of the solution is $\frac{4 \pi p}{n_{l}}$ or $\frac{4 \pi q}{n_{g}}$.

\section{Existence of Periodic Orbits When $\mu \neq 0$}

Here we shall follow the method given by Chaudhary [9] to prove the existence of periodic orbits. Let $x_{1}=L, x_{2}=G, y_{1}=l, y_{2}=g$ then from Equation (44), when $\mu=0$, we have

$$
\begin{aligned}
& \frac{\mathrm{d} x_{i}}{\mathrm{~d} \tau}=0,(i=1,2) \\
& \frac{\mathrm{d} y_{i}}{\mathrm{~d} \tau}=\eta_{i}(0) \text {, say }(i=1,2) \\
& \text { where } \eta_{i}(0)=\left[-2\left(x_{2}+C_{0}\right)\right]^{\frac{1}{2}} ; \eta_{2}(0)=-\frac{x_{1}}{\left[-2\left(x_{2}+C_{0}\right)\right]^{\frac{1}{2}}} .
\end{aligned}
$$

Integrating these equations with respect to $\tau$, we get

$$
x_{i}(0)=a_{i}, \quad y_{i}(0)=\eta_{i}(0) \tau+\omega_{i} .
$$

These are the generating solutions of two-body problems. The generating solution will be periodic with the period $\tau_{0}$, if

$$
y_{i}\left(\tau_{0}\right)-y_{i}(0)=\eta_{i}(0) \tau=2 \pi \kappa_{i}, \quad x_{i}\left(\tau_{0}\right)-x_{i}(0)=0, \quad(i=1,2)
$$

when $\kappa_{i}$ are integers, so that $\eta_{i}(0)$ are commensurable.

Following Poincare [5], the general solution in the neighbourhood of the generating solution, may be given as

$$
x_{i}=a_{i}+\beta_{i}+q_{i}(\varsigma), \quad y_{i}=\eta_{i}(0) \varsigma+\omega_{i}+\gamma_{i}+\eta_{i}(\varsigma), \quad(i=1,2)
$$

where $\varsigma$ is the new independent variable given by

$$
\varsigma=\frac{\tau}{1+\alpha} \text {. }
$$

The necessary and sufficient conditions for the existence of periodic solution are

$$
x_{i}\left(\tau_{0}\right)-x_{i}(0)=q_{i}\left(\tau_{0}\right)=0, \quad y_{i}\left(\tau_{0}\right)-y_{i}(0)=2 \pi \kappa_{i}=\eta_{i}\left(\tau_{0}\right)=0 .
$$

Restricting our solution only up to the first order infinitesimals, the equations of motion may be written as

$$
\begin{gathered}
\frac{\mathrm{d} q_{i}}{\mathrm{~d} \varsigma}=-(1+\alpha) \frac{\partial K}{\partial \omega_{i}}, \\
\frac{\mathrm{d} \eta_{i}}{\mathrm{~d} \varsigma}=(1+\alpha) \frac{\partial K}{\partial a_{i}}+\eta_{i}(0),
\end{gathered}
$$

where

$$
\begin{gathered}
K=K\left[\varsigma, a_{i}+\beta_{i}+q_{i}, \eta_{i}(0) \varsigma+\omega_{i}+\gamma_{i}+\eta_{i}(\varsigma)\right], \\
K=\sum_{i=1}^{2}\left(q_{i} \frac{\partial K}{\partial a_{i}}+\eta_{i} \frac{\partial K}{\partial \omega_{i}}\right)+\eta_{i}(0) q_{i} .
\end{gathered}
$$

Expanding $K\left(\varsigma, a_{i}+\beta_{i}, \eta_{i}(0) \varsigma+\omega_{i}+\gamma_{i}\right)$ in ascending powers of $\beta_{i}, \gamma_{i}, \mu$, 
Equation (54) may be written as

$$
\begin{aligned}
\frac{\mathrm{d} q_{k}}{\mathrm{~d} \varsigma} & =-(1+\alpha) \frac{\partial}{\partial \omega_{k}}\left[K\left(\varsigma, a_{i}+\beta_{i}, \eta_{i}(0) \varsigma+\omega_{i}+\gamma_{i}\right)\right] \\
& =-(1+\alpha) \frac{\partial}{\partial \omega_{K}}\left[K_{0}\left(\varsigma, a_{i}+\beta_{i}\right)+\mu K_{1}\left(\varsigma, a_{i}+\beta_{i}, \eta_{i}(0) \varsigma+\omega_{i}+\gamma_{i}\right)\right] \\
& =-(1+\alpha) \mu \frac{\partial}{\partial \omega_{K}}\left[K_{1}\left(\varsigma, a_{i}, \eta_{i}(0) \varsigma+\omega_{i}\right)+\sum_{i=1}^{2}\left(\beta_{i} \frac{\partial K_{i}}{\partial a_{i}}+\gamma_{i} \frac{\partial K_{i}}{\partial \omega_{i}}\right)\right] .
\end{aligned}
$$

Rejecting the second order term $\alpha \mu$, integrating and putting the value of $q_{1}$ in Equation (51), we get

$$
\frac{q_{k}\left(\tau_{0}, \beta_{i}, \gamma_{i}\right)}{-\mu \tau_{0}}=\frac{\partial\left[K_{1}\right]}{\partial \omega_{K}}+\sum_{i=1}^{2}\left[\beta_{i} \frac{\partial^{2}\left[K_{1}\right]}{\partial \omega_{K} \partial a_{i}}+\gamma_{i} \frac{\partial^{2}\left[K_{1}\right]}{\partial \omega_{K} \partial \omega_{i}}\right] \text {, where } k=1,2
$$

and $\left[K_{1}\right]=\frac{1}{\tau_{0}} \int_{0}^{\tau_{0}} K_{1}\left\{\xi_{1}, a_{i}, \eta_{i}(0) \varsigma+\omega_{i}\right\} \mathrm{d} \varsigma$.

The Equation (55) gives

$$
\begin{aligned}
& \frac{\mathrm{d} \eta_{1}}{\mathrm{~d} \varsigma}= \eta_{1}(0)+(1+\alpha) \frac{\partial}{\partial a_{1}}\left[K_{0}\left(\varsigma, a_{i}+\beta_{i}, \eta_{i}(0) \varsigma+\omega_{i}+\gamma_{i}\right)\right]_{(0)} \\
&= \eta_{1}(0)+(1+\alpha) \frac{\partial}{\partial a_{1}}\left[K_{0}\left(\varsigma, a_{i}+\beta_{i}\right)+\mu K_{1}\left(\varsigma, a_{i}+\beta_{i}, \eta_{i}(0) \varsigma+\omega_{i}+\gamma_{i}\right)\right] \\
&= \eta_{1}(0)+(1+\alpha) \frac{\partial}{\partial a_{1}}\left[K_{0}\left(\varsigma, a_{i}\right)+\sum_{i=1}^{2} \beta_{i} \frac{\partial K_{0}}{\partial a_{i}}\right. \\
&\left.+\mu\left\{K_{1}\left(\varsigma, a_{i}, \eta_{i}(0) \varsigma+\omega_{i}\right)+\sum_{i=1}^{2}\left(\beta_{i} \frac{\partial K_{1}}{\partial a_{1}}+\gamma \frac{\partial K_{1}}{\partial \omega_{i}}\right)\right\}\right] \\
&= \eta_{1}(0)+(1+\alpha) \frac{\partial K_{0}}{\partial a_{1}}+\sum_{i=1}^{2} \beta_{i} \frac{\partial^{2} K_{0}}{\partial \omega_{1} \partial a_{i}}+O(\mu), \\
& \frac{\mathrm{d} \eta_{1}}{\mathrm{~d} \varsigma}=\alpha \frac{\partial K_{0}}{\partial a_{1}}+\beta_{1} \frac{\partial^{2} K_{0}}{\partial a_{1}^{2}}+\beta_{2} \frac{\partial^{2} K_{0}}{\partial a_{1} \partial a_{2}}+O(\mu) . \quad\left[\because \eta_{1}(0)=-\frac{\partial K_{0}}{\partial a_{1}}\right]
\end{aligned}
$$

Equation (45) gives

$$
\left.\begin{array}{l}
\eta_{1}\left(\tau_{0}, \beta_{i}, \gamma_{i}, \mu\right)=\alpha \tau_{0} \frac{\partial K_{0}}{\partial a_{1}}+\beta_{1} \tau_{0} \frac{\partial^{2} K_{0}}{\partial a_{1}^{2}}+\beta_{2} \tau_{0} \frac{\partial^{2} K_{0}}{\partial a_{1} \partial a_{2}}+O(\mu), \\
\eta_{2}\left(\tau_{0}, \beta_{i}, \gamma_{i}, \mu\right)=\alpha \tau_{0} \frac{\partial K_{0}}{\partial a_{2}}+\beta_{1} \tau_{0} \frac{\partial^{2} K_{0}}{\partial a_{1} a_{2}}+\beta_{2} \tau_{0} \frac{\partial^{2} K_{0}}{\partial a_{2}^{2}}+O(\mu) .
\end{array}\right\}
$$

By solving the Equations (54)-(57), we can find the values of $\beta_{1}, \beta_{2}, \gamma_{2}$, as analytic function of $\mu$, reducing to zero with $\mu$, if the conditions for periodic orbits given by Duboshin [4] are satisfied i.e.,

(i) $\frac{\partial\left[K_{1}\right]}{\partial \omega_{i}}=0, i=1,2$, and

(ii) $\frac{\partial\left[K_{1}\right]}{\partial a_{i}}=0, i=1,2$, together

(iii) $\frac{\partial\left(q_{2}, \eta_{1}, \eta_{2}\right)}{\partial\left(\gamma_{2}, \beta_{1}, \beta_{2}\right)} \neq 0$ for $\mu=\beta_{i}=\gamma_{i}=0$, 
where $\left[K_{1}\right]$ is the zero degree terms of $K_{1}$ given in Equation (26).

Now,

$$
\frac{\partial\left(q_{2}, \eta_{1}, \eta_{2}\right)}{\partial\left(\gamma_{2}, \beta_{1}, \beta_{2}\right)}=\left|\begin{array}{lll}
\frac{\partial q_{2}}{\partial \gamma_{2}} & \frac{\partial \eta_{2}}{\partial \gamma_{2}} & \frac{\partial \eta_{2}}{\partial \gamma_{2}} \\
\frac{\partial q_{2}}{\partial \beta_{1}} & \frac{\partial \eta_{1}}{\partial \beta_{1}} & \frac{\partial \eta_{2}}{\partial \beta_{1}} \\
\frac{\partial q_{2}}{\partial \beta_{2}} & \frac{\partial \eta_{1}}{\partial \beta_{2}} & \frac{\partial \eta_{2}}{\partial \beta_{2}}
\end{array}\right|=\left|\begin{array}{ccc}
\frac{\partial^{2}\left[K_{1}\right]}{\partial \omega_{2}^{2}} & 0 & 0 \\
\frac{\partial^{2}\left[K_{1}\right]}{\partial \omega_{2} \partial a_{1}} & \tau_{0} \frac{\partial^{2} K_{0}}{\partial a_{1}^{2}} & \tau_{0} \frac{\partial^{2} K_{0}}{\partial a_{1} \partial a_{2}} \\
\frac{\partial^{2}\left[K_{1}\right]}{\partial \omega_{2} \partial a_{2}} & \tau_{0} \frac{\partial^{2} K_{0}}{\partial a_{1} \partial a_{2}} & \tau_{0} \frac{\partial^{2} K_{0}}{\partial a_{2}^{2}}
\end{array}\right|,
$$

From Equation (43),

$$
\begin{aligned}
K_{0} & =L\left[-2\left(G+C_{0}\right)\right]^{\frac{1}{2}}-1=x_{1}\left[-2\left(x_{2}+C_{0}\right)\right]^{\frac{1}{2}}-1 \\
& =a_{1}\left[-2\left(a_{2}+C_{0}\right)\right]^{\frac{1}{2}}-1,
\end{aligned}
$$

then

$$
\left.\begin{array}{rl} 
& \frac{\partial K_{0}}{\partial a_{1}}=\left[-2\left(a_{2}+C_{0}\right)\right]^{\frac{1}{2}}, \\
\Rightarrow & \frac{\partial^{2} K_{0}}{\partial a_{1} \partial a_{2}}=\frac{-a_{2}}{\left[-2\left(a_{2}+C_{0}\right)\right]^{\frac{1}{2}}} \text { and } \frac{\partial^{2} K_{0}}{\partial a_{1}^{2}}=0 .
\end{array}\right\}
$$

From the Equation (26)

$$
K_{1}=1-\rho^{2} C_{1}-\frac{1}{2}\left(Q_{1} q_{2}+Q_{2} q_{1}\right)-\frac{\rho^{2}}{r_{2}}-\varepsilon_{0} \frac{\rho^{2}}{r_{3}},
$$

where

$$
\begin{gathered}
\rho^{2}=z=a(1-e \cos l)=r_{1}, \\
r_{2}^{2}=1+2 a(1-e \cos l) \cos 2 \varphi+a^{2}(1-e \cos l)^{2}, \\
r_{3}^{2}=1+2 a(1-e \cos l) \cos \left(2 \varphi+\frac{\pi}{3}\right)+a^{2}(1-e \cos l)^{2}, \\
Q_{1} q_{2}+Q_{2} q_{1}=2(e L \sin l \sin 2 \varphi+\varsigma \cos 2 \varphi),
\end{gathered}
$$

Thus

$$
K_{1}=1-C_{1} a(1-e \cos l)-e L \sin l \sin 2 \varphi-G \cos 2 \varphi-a(1-e \cos l)\left(\frac{1}{r_{2}}+\frac{\varepsilon_{0}}{r_{3}}\right) .
$$

Taking only zero order terms i.e., for $e=0, h=l=y_{1}$

$$
\left.\begin{array}{l}
r_{1}=\rho^{2}=z=a, \\
r_{2}^{2}=1+2 a \cos 2 \varphi+a^{2}, \\
r_{3}^{2}=1+2 a \cos \left(2 \varphi+\frac{\pi}{3}\right)+a^{2}, \\
{\left[K_{1}\right]=1-C_{1} a-G \cos 2 \varphi-a\left(\frac{1}{r_{2}}+\frac{\varepsilon_{0}}{r_{3}}\right),}
\end{array}\right\}
$$

where $2 \varphi=y_{1}+y_{2}-\frac{\sqrt{x_{1}^{2}-x_{2}^{2}}}{2\left(x_{2}+C_{0}\right)} \sin y_{1}$. 
Now from equations of system (52)

$$
\left.\begin{array}{ll}
x_{1}=a_{1}+\beta_{1}+q_{1}(\varsigma), & x_{2}=a_{2}+\beta_{2}+q_{2}(\varsigma), \\
y_{1}=\eta_{1}(0) \varsigma+\omega_{1}+\gamma_{1}+\eta_{1}(\varsigma), & y_{2}=\eta_{2}(0) \varsigma+\omega_{2}+\gamma_{2}+\eta_{2}(\varsigma) .
\end{array}\right\}
$$

and from Equation (63)

$$
\begin{aligned}
& \frac{\partial\left[K_{1}\right]}{\partial a_{1}}=\frac{N a_{1} \sin y_{1}}{2\left(a_{2}+C_{0}\right) \sqrt{a_{1}^{2}-a_{2}^{2}}}, \\
& \frac{\partial\left[K_{1}\right]}{\partial a_{2}}=\frac{N\left(a_{1}^{2}+a_{2} C_{0}\right) \sin y_{1}}{2\left(a_{2}+C_{0}\right)^{2}}, \\
& \frac{\partial\left[K_{1}\right]}{\partial \omega_{1}}=M N=\frac{\partial\left[K_{1}\right]}{\partial \omega_{2}},
\end{aligned}
$$

where

$$
M=\frac{\sqrt{a_{1}^{2}-a_{2}^{2}} \cos y_{1}}{2\left(a_{2}+C_{0}\right)}-1 \text { and } N=\left(\frac{a^{2}}{r_{2}^{3}}+\frac{a^{2} \varepsilon_{0}}{2 r_{3}^{3}}-G\right) \sin 2 \varphi+\frac{\sqrt{3} a^{2} \varepsilon_{0}}{2 r_{3}^{3}} \cos 2 \varphi .
$$

Here $\frac{\partial\left[K_{1}\right]}{\partial a_{i}}=0$ if either $\sin y_{1}=0$ or $N=0$ and $\frac{\partial\left[K_{1}\right]}{\partial \omega_{i}}=0$ if either $M=0$ or $M=0$.

But $\sin y_{1}=0$ and $M=\frac{\sqrt{a_{1}^{2}-a_{2}^{2}}}{2\left(a_{2}+C_{0}\right)} \cos y_{1}-1=0$ don't imply each other, so $N=0$ is only the case for which $\frac{\partial\left[K_{1}\right]}{\partial a_{i}}$ and $\frac{\partial\left[K_{1}\right]}{\partial \omega_{i}}$ will be simultaneously zero.

Now choosing suitably $2 \varphi=\frac{5 \pi}{6}$, then $r_{2}=r_{3}=\xi$ (say) and

$$
\begin{aligned}
& N=\left(\frac{a^{2}}{\xi^{3}}+\frac{a^{2} \varepsilon_{0}}{2 \xi^{3}}-G\right) \sin \frac{5 \pi}{6}+\frac{\sqrt{3} a^{2} \varepsilon_{0}}{2 \xi^{3}} \cos \frac{5 \pi}{6}, \\
& N=\left(\frac{a^{2}}{\xi^{3}}+\frac{a^{2} \varepsilon_{0}}{2 \xi^{3}}-G\right) \frac{1}{2}+\frac{\sqrt{3} a^{2} \varepsilon_{0}}{2 \xi^{3}}\left(-\frac{\sqrt{3}}{2}\right), \\
& N=\frac{1}{2}\left[\frac{a^{2}}{\xi^{3}}+\frac{a^{2} \varepsilon_{0}}{2 \xi^{3}}-G-\frac{3 a^{2} \varepsilon_{0}}{2 \xi^{3}}\right], \\
& \text { i.e., } N=\frac{1}{2}\left[\frac{a^{2}}{2 \xi^{3}}\left(1-\varepsilon_{0}\right)-G\right] .
\end{aligned}
$$

Thus,

$$
\frac{\partial\left[K_{1}\right]}{\partial a_{i}}=\frac{\partial\left[K_{1}\right]}{\partial \omega_{i}}=0, \text { if } 2 \varphi=\frac{5 \pi}{6} \text { and } G=\frac{a^{2}}{\xi^{3}}\left(1-\varepsilon_{0}\right) .
$$

Now,

$$
\frac{\partial\left[K_{1}\right]}{\partial \omega_{2}}=\left(\frac{\sqrt{a_{1}^{2}-a_{2}^{2}}}{2\left(a_{2}+C_{0}\right)} \cos y_{1}-1\right)\left[\left(\frac{a^{2}}{r_{2}^{3}}+\frac{a^{2} \varepsilon_{0}}{2 r_{3}^{3}}-G\right) \sin 2 \varphi+\frac{\sqrt{3} a^{2} \varepsilon_{0}}{2 r_{3}^{3}} \cos 2 \varphi\right],
$$




$$
\begin{aligned}
\Rightarrow \frac{\partial^{2}\left[K_{1}\right]}{\partial \omega_{2}^{2}}= & M\left[-\frac{3 a^{2} \sin 2 \varphi}{r_{2}^{4}} \frac{\partial r_{2}}{\partial \omega_{2}}-\frac{\sqrt{3} a^{2} \varepsilon_{0}}{2 r_{2}^{3}} \sin 2 \varphi 2 \frac{\partial \varphi}{\partial \omega_{2}}\right. \\
& \left.-\frac{3 a^{2} \varepsilon_{0}}{2 r_{3}^{4}}(\sin 2 \varphi+\sqrt{3} \cos 2 \varphi) \frac{\partial r_{3}}{\partial \omega_{2}}\left(\frac{a^{2}}{r_{2}^{3}}+\frac{a^{2} \varepsilon_{0}}{2 r_{3}^{3}}-G\right) \cos 2 \varphi 2 \frac{\partial \varphi}{\partial \omega_{2}}\right] .
\end{aligned}
$$

As $2 \frac{\partial \varphi}{\partial \omega_{2}}=1$ so from Equation (63), we have

$$
\begin{aligned}
& \frac{\partial r_{2}}{\partial \omega_{2}}=-\frac{a \sin 2 \varphi}{r_{2}}, \\
& \frac{\partial r_{3}}{\partial \omega_{2}}=-\frac{a \sin \left(2 \varphi+\frac{\pi}{3}\right)}{r_{3}},
\end{aligned}
$$

Thus,

$$
\begin{aligned}
\frac{\partial^{2}\left[K_{1}\right]}{\partial \omega_{2}^{2}}= & M\left[\left(\frac{a^{2}}{r_{2}^{3}}+\frac{a^{2} \varepsilon_{0}}{2 r_{3}^{3}}-G\right) \cos 2 \varphi-\frac{\sqrt{3} a^{2} \varepsilon_{0}}{2 r_{2}^{3}} \sin 2 \varphi-\frac{\sqrt{3} a^{2} \varepsilon_{0}}{2 r_{3}^{3}} \sin 2 \varphi\right. \\
& \left.+\frac{3 a^{3}}{r_{2}^{5}} \sin ^{2} 2 \varphi+\frac{3 a^{3} \varepsilon_{0}}{r_{2}^{5}} \sin \left(2 \varphi+\frac{\pi}{3}\right)\right] .
\end{aligned}
$$

Using Equation (65), we get

$$
\begin{gathered}
\frac{\partial^{2}\left[K_{1}\right]}{\partial \omega_{2}^{2}}=\frac{M \sqrt{3} a^{2}}{\xi^{3}}\left[\frac{\sqrt{3} a\left(1-\varepsilon_{0}\right)}{4 \xi^{2}}-\varepsilon_{0}\right],\left(\text { as } \varepsilon_{0} \ll 1\right) \\
\Rightarrow \frac{\partial^{2}\left[K_{1}\right]}{\partial \omega_{2}^{2}} \neq 0, \\
\therefore \frac{\partial\left(q_{2}, \eta_{1}, \eta_{2}\right)}{\partial\left(\gamma_{1}, \beta_{1}, \beta_{2}\right)} \neq 0 .
\end{gathered}
$$

Thus the conditions for the existence of periodic orbits given by Duboshin [4] are satisfied i.e., in the region of motion of the infinitesimal body, periodic orbits exist.

\section{Poincare Surfaces of Section (PSS)}

In this previous section, we have shown that Duboshin's condition [4] for the existence of periodic orbits when $\mu \neq 0$, are satisfied. So to justify the mathematical model given in Equations (58)-(60), we have applied the method of Poincare surfaces of section (PSS) to the reduced equations of motion

$$
\left.\begin{array}{l}
\ddot{x}+2 \dot{y}=x+\frac{(1-\mu)(x-\mu)}{r_{1}^{3}}+\frac{\mu(x-\mu+1)}{r_{2}^{3}}+\frac{\mu \varepsilon_{0}\left(x-\mu+\frac{1}{2}\right)}{r_{3}^{3}}, \\
\ddot{y}+2 \dot{x}=y+\frac{(1-\mu) y}{r_{1}^{3}}+\frac{\mu y}{r_{2}^{3}}+\frac{\mu \varepsilon_{0}\left(y-\frac{\sqrt{3}}{2}\right)}{r_{3}^{3}},
\end{array}\right\}
$$

together with the Jacobi Integral

$$
\dot{x}^{2}+\dot{y}^{2}-\left(x^{2}+y^{2}\right)+2\left[\frac{1-\mu}{r_{1}}+\frac{\mu}{r_{2}}+\frac{\varepsilon}{r_{3}}\right]=C .
$$

To study the motion of the infinitesimal body by PSS, it is necessary to know 
its position $(x, y)$ and velocity $(\dot{x}, \dot{y})$ which correspond to a point in fourdimensional phase space. By defining a plane $y=0$, in the resulting three- dimensional space, the values of $x$ and $\dot{x}$ can be plotted. Every time the particle has $y=0$, whenever the trajectory intersects the plane in a particular direction say $\dot{y}>0$.

The techniques of PSS suggest to determine the regular or chaotic nature of the trajectories. If there are smooth, well-defined island then the trajectory is likely to be regular and the islands correspond to oscillation around a periodic orbit. As the curves shrink down to a point, the points represent a periodic orbit as per Kolmogorov-Arnold-Moser (KAM) theory. Any fuzzy distribution of points in surfaces of section, implies that trajectory is chaotic. In Figure 2, for $C=3.15, \mu=0.0125$ and $\varepsilon=0.000001$ Poincare surfaces of section have been plotted in which atleast seven points are visible towards which the regular trajectories shrink, hence by KAM theory, periodic orbits exists. Again Figure 3 represents a Poincare surfaces of section for $C=3.17, \mu=0.0125$ and $\varepsilon=0.000001$ in which atleast nine points are visible towards which the regular trajectories shrink, so we can say that the periodic orbits exist in the region of motion of infinitesimal mass. Other than the neighbourhood of these points, the quasi-periodic and chaotic regions are seen in the PSS. In Figure 4, in PSS for $C=3.17, \mu=0.0125$ and $\varepsilon=0.000001$, atleast ten shrinking regions of regular curves to a point are visible, i.e., that the degree of existence of periodic orbits increases in the region of motion of the infinitesimal mass. Thus by increasing the values of the Jacobi's constant, the chances of existence of periodic orbits increase. Thus the Duboshin conditions and PSS both confirms the existence of periodic orbits when $\mu \neq 0$. In Figure 5, regions plot of ZVC (Zero Velocity Curves) for $C=3.172$ is shown, in which central white circle represents regions of no motion and coloured annulus represents the regions of periodic orbits. Figure 6 depicts the contour plot of ZVC for $C=3.172$.

\section{Periodic Orbits of Collision When $\mu \neq 0$}

Levi-Civita [6] [7] proved that the invariant relation for collision orbits can be analytically continued from the one that corresponds to the problem of two bo-

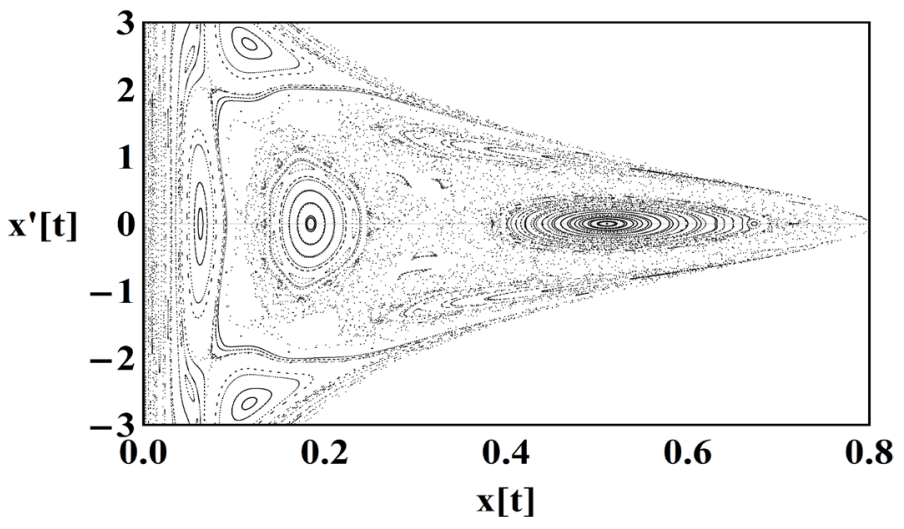

Figure 2. Poincare Surface of Section for $C=3.15, \mu=0.0125$ and $\varepsilon=0.000001$. 


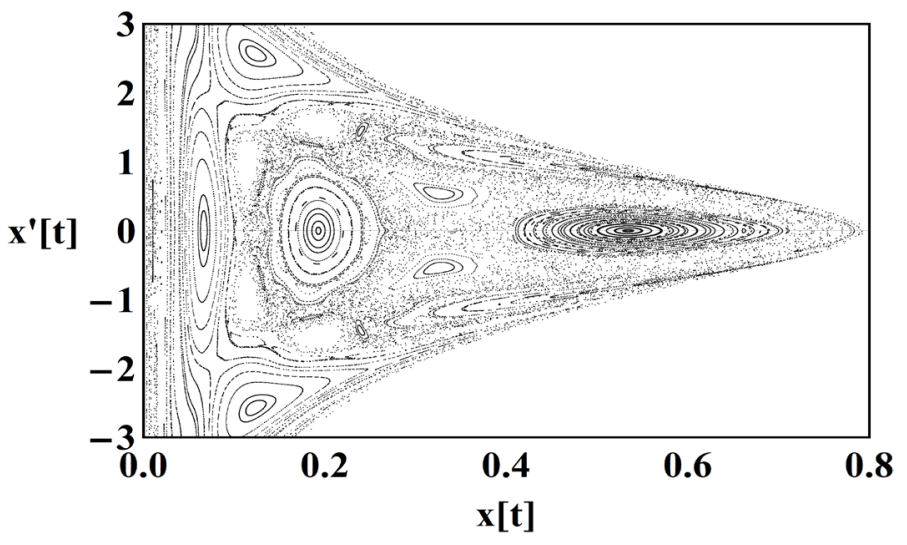

Figure 3. Poincare Surface of Section for $C=3.17, \mu=0.0125$ and $\varepsilon=0.000001$.

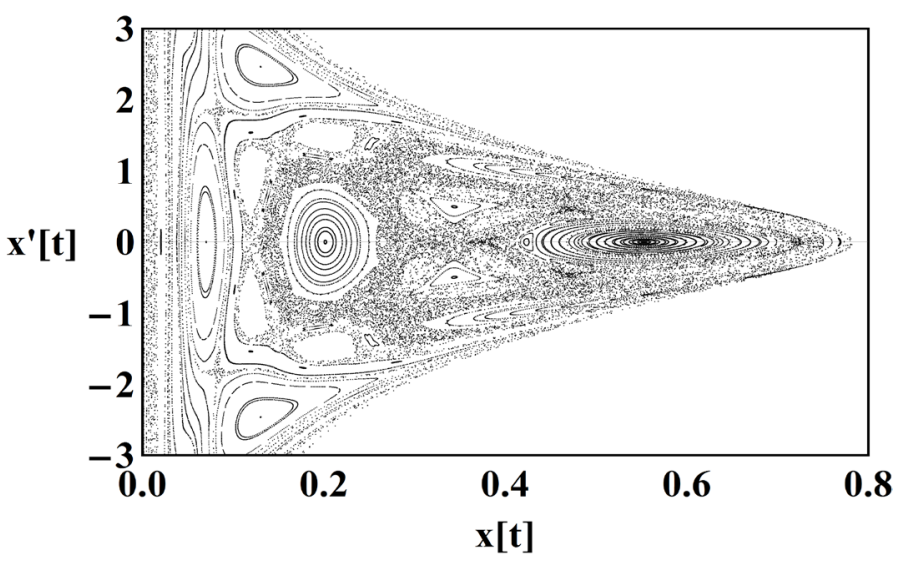

Figure 4. Poincare Surface of Section for $C=3.19, \mu=0.0125$ and $\varepsilon=0.000001$.

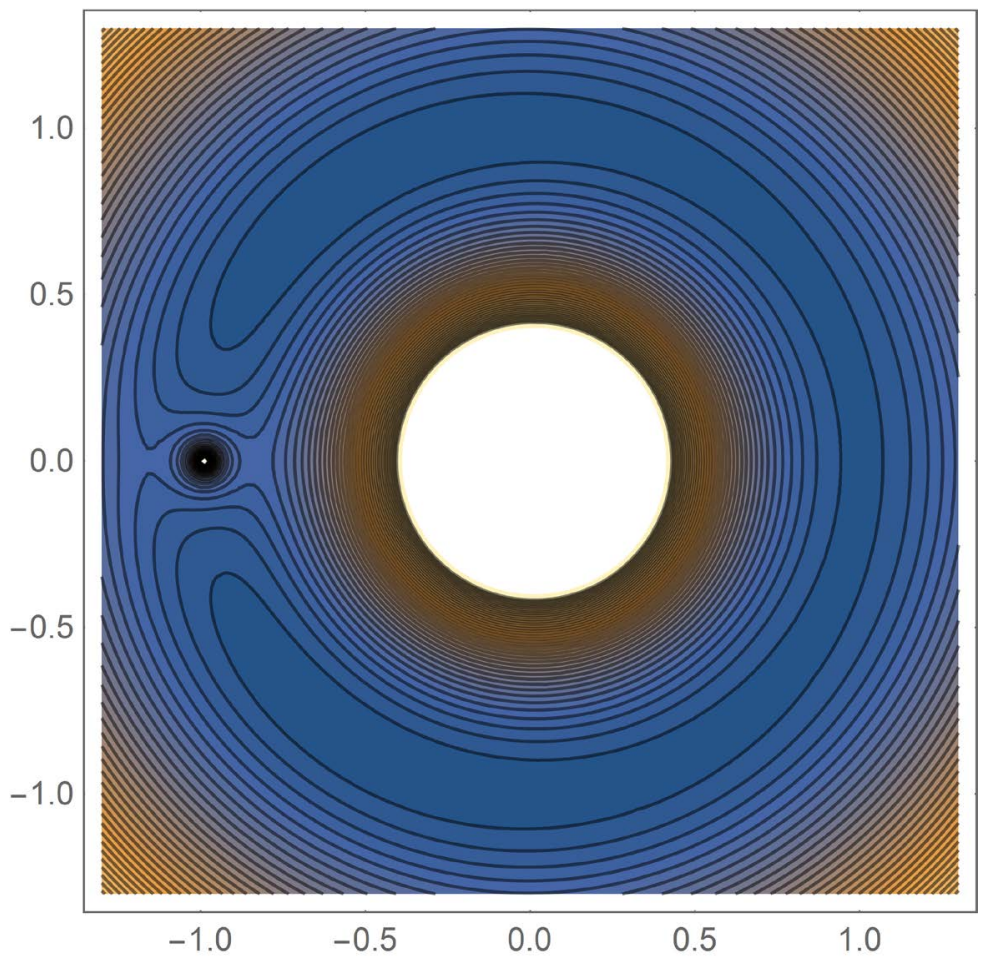

Figure 5. Region Plot of ZVCs for $C=3.172$. 


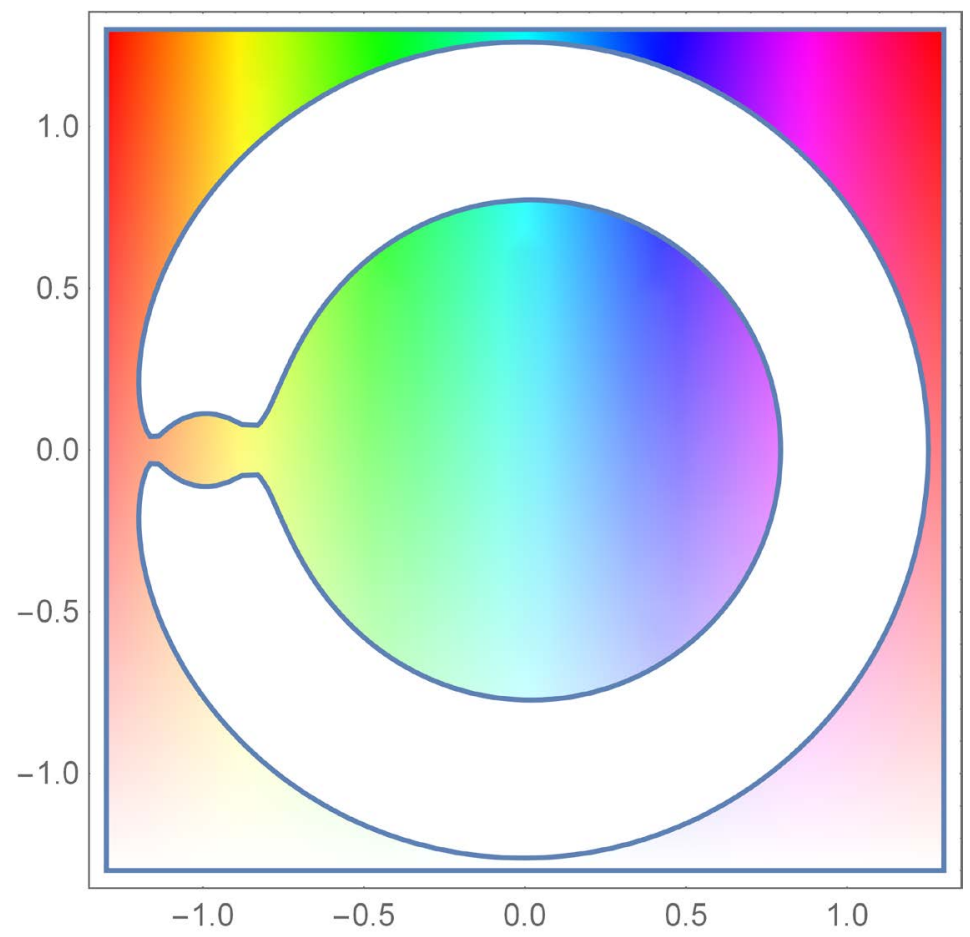

Figure 6. Contour Plot of ZVCs for $C=3.172$.

dies. Bhatnagar [2] [3] has developed this as $G=0$. For the present paper when $\mu \neq 0$, the condition must be

$$
G+\mu F\left(l, L, g, G, \mu, \varepsilon_{0}\right)=0
$$

for sufficiently small $\mu$ and $\varepsilon_{0}$.

Further, he has proved that, in particular, such relation is uniform integral of the differential equation of motion along any collision orbit. He has also proved this integral is a power series in terms of the distance from the origin and the series is convergent through the radius of convergence is generally small. In section (5), we have shown that periodicity is conserved by analytic continuation. Let us show that the condition of collision is also conserved by analytic continuation.

Figure 7 shows the geometrical configuration of collision orbits. In order to show the validity of that continuation, we shall consider orbits corresponding to the case when $e=1$ (i.e., $G=0$ ). When $e=1$, the orbits starts as an ejection from the origin and return to it after $\frac{T}{4}$. Bhatnagar [2] [3] and Levi-Civita [6] [7] finds the condition for collision as

$$
\dot{\theta}+1=\xi f(\xi, \theta)
$$

where

$$
\begin{aligned}
\tan \theta=\frac{x_{2}}{x_{1}-\mu}, \quad \xi=\sqrt{r_{1}}, \\
=\frac{2 q_{1} q_{2}}{q_{1}^{2}-q_{2}^{2}}=\frac{2 \rho \cos \varphi \cdot \rho \sin \varphi}{\rho^{2} \cos ^{2} \varphi-\rho^{2} \sin ^{2} \varphi}=\tan 2 \varphi, \\
\quad \Rightarrow \theta=2 \varphi .
\end{aligned}
$$




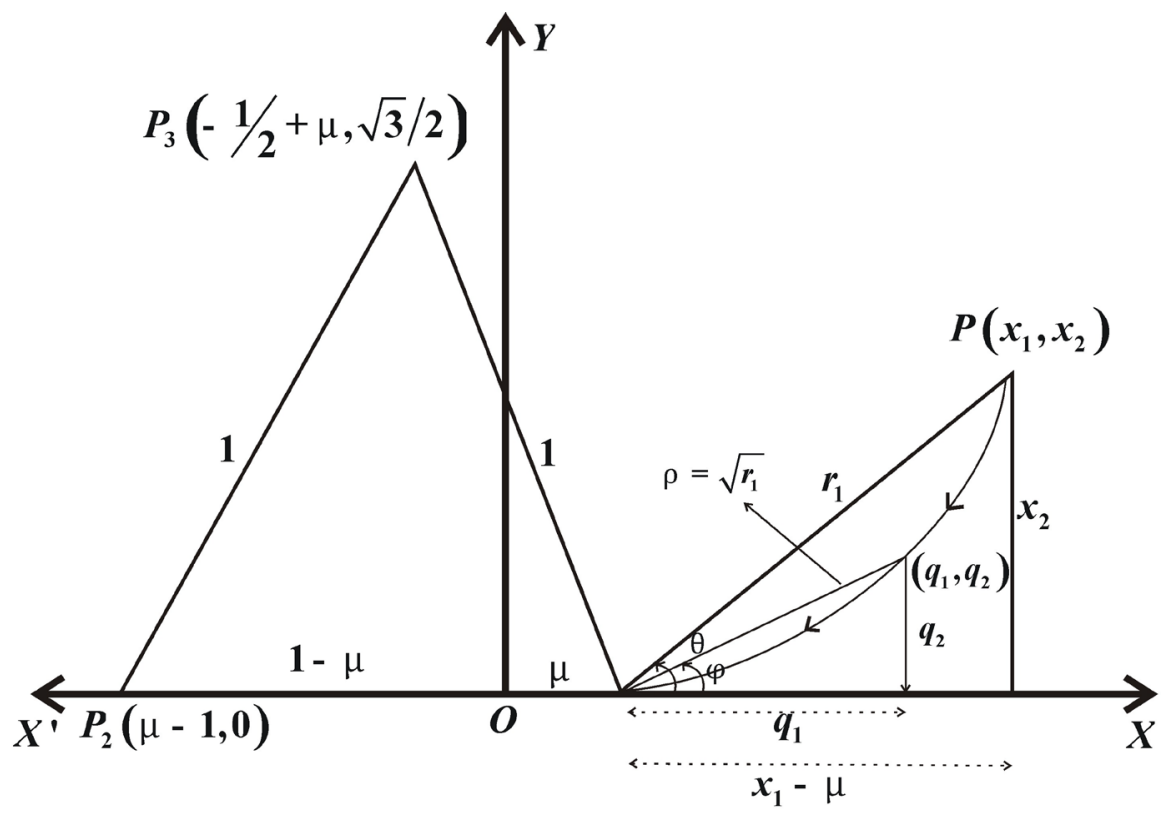

Figure 7. Geometrical configuration of collision orbits.

Therefore, the condition of Equation (69) became,

$$
\begin{aligned}
& 2 \dot{\varphi}+1=\sqrt{r_{1}} f\left(\sqrt{r_{1}}, 2 \varphi\right), \\
& 2 \frac{\mathrm{d} \varphi}{\mathrm{d} t}+1=\sqrt{r_{1}} f\left(\sqrt{r_{1}}, 2 \varphi\right), \\
& 2 \frac{\mathrm{d} \varphi}{\mathrm{d} t}+r_{1}=r^{\frac{3}{2}} f\left(\sqrt{r_{1}}, 2 \varphi\right) .
\end{aligned}
$$

But,

$$
\begin{gathered}
\tan \varphi=\frac{q_{2}}{q_{1}} \text { so } \varphi=\tan ^{-1}\left(\frac{q_{2}}{q_{1}}\right), \\
\therefore \frac{\mathrm{d} \varphi}{\mathrm{d} \tau}=\frac{1}{1+\frac{q_{2}^{2}}{q_{1}^{2}}} \times \frac{q_{1} q_{2}^{\prime}-q_{2} q_{1}^{\prime}}{q_{1}^{2}}, \\
\frac{\mathrm{d} \varphi}{\mathrm{d} \tau}=\frac{q_{1} q_{2}^{\prime}-q_{2} q_{1}^{\prime}}{q_{1}^{2}+q_{2}^{2}}, \\
\frac{\mathrm{d} \varphi}{\mathrm{d} \tau}=\frac{\left(q_{1} Q_{2}-q_{2} Q_{1}\right)}{4 \rho^{2}}-\frac{1}{2} \rho^{2}=\frac{G}{2 r_{1}}-\frac{r_{1}}{2} .
\end{gathered}
$$

Thus from Equations ((70) and (71))

$$
\begin{gathered}
2\left(\frac{G}{2 r_{1}}-\frac{r_{1}}{2}\right)+r_{1}=r_{1}^{\frac{3}{2}} f\left(\sqrt{r_{1}}, 2 \varphi\right), \\
G-r_{1}^{\frac{5}{2}} f\left(\sqrt{r_{1}}, 2 \varphi\right)=0, \\
G-\xi^{5} f(\xi, 2 \varphi)=0 .
\end{gathered}
$$

Here the Equation (71) corresponds to the Equation (68), so it is easy to say that the collision orbits exist. 


\section{Discussions and Conclusion}

In section 1 of this paper, historical background has been sketched with original and previous contributions. In section 2 , the equations of motion of the infinitesimal mass moving under the gravitational field of the three primaries situated at the vertices of an equilateral triangle taken by Ceccaroni and Biggs [8]. In this the reduced Hamiltonian $H=C$ has been derived for regularization in the next section 3. In this section, the regularized Hamiltonian $K=0$ has been established. In section 4 , generating solutions have been found by taking $K_{0}$ as the corresponding Hamiltonian. In this section generating solution forms a basis for general solution by the process of analytic continuation. In section 5, using Duboshin's criterion [4] for the existence of periodic orbits has been satisfied following the method of Choudhary [9]. For confirmation of the existence of periodic orbits in section 4, we have analyzed PSS in section 6 and justified that the region of motion regular trajectory shrinking towards a point represents the periodic orbits and other region of the PSS represents quasi-periodic and chaotic belt in the region of motion. In section 7 , the periodic orbits of collision for $\mu \neq 0$ have been shown. In our discussion, we have shown that our condition of collision orbit $G+\mu F\left(l, L, g, G, \mu, \varepsilon_{0}\right)=0$ has a resemblance with the condition given by Bhatnagar [2] [3].

\section{References}

[1] Giacaglia, E.O. (1967) Periodic Orbits of Collision in the Restricted Problem of Three Bodies. Astronomical Journal, 72, 386-391. https://doi.org/10.1086/110237

[2] Bhatnagar, K.B. (1969) Periodic Orbits of Collision in the Plane Elliptic Restricted Problem of Three Bodies. National Institute of Science India, 35A, 829-844.

[3] Bhatnagar, K.B. (1971) Periodic Orbits of Collision in the Plane Circular Problem of Four Bodies. Indian Journal of Pure and Applied Mathematics, 2, 583-596.

[4] Duboshin, G.N. (1964) Analytical and Qualitative Methods (Russian). Celestial Mechanics, 178-184.

[5] Poincare, H. (1905) Lecons de Mécanique Céleste. Gauthier-Villars, Paris, 1.

[6] Levi-Civita, T. (1903) Traiettorie singolari ed urti nel problema ristreto deri teri corpi. Annali di Mathematica Pura ed Applicata, 9, 1-32.

[7] Levi-Civita, T. (1906) Sur la résolution qualitative du probleme restrient des trios corps. Acta Mathematica, 30, 305-327. https://doi.org/10.1007/BF02418577

[8] Ceccaroni, M. and Biggs, J. (2012) Low-Thrust Propulsion in a Coplanar Circular Restricted Four body Problem. Celestial Mechanics and Dynamical Astronomy, 112, 191-219. https://doi.org/10.1007/s10569-011-9391-x

[9] Choudhry, R.K. (1966) Existence of Periodic Orbits of the Third Kind in the Elliptic Restricted Problem of the Three Bodies and the Stability of the Generating Solution. Proceedings of the National Academy of Sciences India Section A, 36, 249-264. 


\section{Definitions}

Bary-Centre: It is the center of mass of two or more bodies that are orbiting each other, or the point around which they both orbit.

Synodic Co-ordinate System: The co-ordinate system, in which the xy-plane rotates in the positive direction with an angular velocity equal to that of the common velocity of one primary with respect to the other keeping the origin fixed, is called synodic co-ordinate system.

Reduced Mass: Mass ratio of the smaller primary to the total mass of the primaries or the non-dimensional mass of the smaller primary is known as reduced mass of the smaller primary.

Regularization: The process of elimination of the singularity from the force function is known as regularization.

Submit or recommend next manuscript to SCIRP and we will provide best service for you:

Accepting pre-submission inquiries through Email, Facebook, LinkedIn, Twitter, etc. A wide selection of journals (inclusive of 9 subjects, more than 200 journals) Providing 24-hour high-quality service User-friendly online submission system Fair and swift peer-review system Efficient typesetting and proofreading procedure Display of the result of downloads and visits, as well as the number of cited articles Maximum dissemination of your research work

Submit your manuscript at: http://papersubmission.scirp.org/

Or contact ijaa@scirp.org 